\title{
Neuron-Specific BAF Complex
}

National Cancer Institute

\section{Source}

National Cancer Institute. Neuron-Specific BAF Complex. NCI Thesaurus. Code C156920.

A SWI/SNF family chromatin-remodeling complex expressed in postmitotic neurons and comprised of at least ACTB, ARID1A/BAF250A, ARID1B/BAF250B or ARID2/BAF200, BCL11A or BCL11B, SMARCA2/BRM/BAF190B or SMARCA4/BRG1/BAF190A, ACT L6B/BAF53B, SMARCB1/BAF47, SMARCC1/BAF155, SMARCE1/BAF57, SMARCC2/BAF170, SS18L1/CREST, PBRM1/BAF180, DPF1/BAF45B or DPF3/BAF45C, BCL7A, BCL7B or BCL7C, and SMARCD1/BAF60A, SMARCD2/BAF60B or SMARCD3/BAF60C. 